\title{
Diagnosis in interstitial lung disease: highly confident histopathological results from transbronchial cryobiopsy are reliable
}

\author{
Lars Hagmeyer ${ }^{1,2}$, Sandhya Matthes ${ }^{2}$, Konrad Frank ${ }^{3}$, Winfried Randerath ${ }^{1,2}$ \\ ${ }^{1}$ Institute of Pneumology, University of Cologne, Solingen, Germany; ${ }^{2}$ Hospital Bethanien Solingen, Clinic of Pneumology and Allergology, \\ Center for Sleep Medicine and Respiratory Care, Solingen, Germany; ${ }^{3}$ University Hospital of Cologne, Section Pneumology, Clinic III of Internal \\ Medicine, Cologne, Germany \\ Correspondence to: Lars Hagmeyer, MD. Hospital Bethanien Solingen, Clinic for Pneumology and Allergology, Center of Sleep Medicine and \\ Respiratory Care, Aufderhöherstraße 169-175, 42699 Solingen, Germany. Email: lars.hagmeyer@klinik-bethanien.de. \\ Comment on: Troy LK, Grainge C, Corte TJ, et al. Diagnostic accuracy of transbronchial lung cryobiopsy for interstitial lung disease diagnosis \\ (COLDICE): a prospective, comparative study. Lancet Respir Med 2020;8:171-81.
}

Submitted Apr 04, 2020. Accepted for publication Apr 28, 2020.

doi: $10.21037 /$ atm-20-3132

View this article at: http://dx.doi.org/10.21037/atm-20-3132

\section{Lung tissue sampling and multidisciplinary discussion in interstitial lung disease (ILD)}

The establishment of a conclusive clinical diagnosis in ILD is essential. In most cases, it is based on the merger of findings from clinical, serological and radiological investigations. High resolution computed tomography (HRCT) has become a very important diagnostic tool, as radiologic findings give an impression of the nature and prognosis of the disease in the vast majority of patients. Bronchoalveolar lavage may be of additive value (1). However, in some cases the clinical diagnosis remains unclear, making lung tissue sampling necessary.

Integrating data from all investigations in a multidisciplinary discussion (MDD) allows for a conclusive diagnosis in most cases $(2,3)$. The IPF guideline emphasizes the benefit of MDD when the HRCT pattern is probable usual interstitial pneumonia (UIP), indeterminate for UIP, or an alternative diagnosis, or when there are discordant clinical, radiologic, and/or histologic data (1).

Historically, surgical lung biopsy (SLB) represents the most frequently performed approach for lung tissue sampling and has been recommended in the guidelines following disappointing results from transbronchial forceps biopsy trials (1).

SLB is associated with a relevant morbidity and mortality (1). In clinical practice, lung tissue sampling very often does not occur, as patients are unwilling to undergo a surgical procedure purely for diagnostic purposes. In other cases, the physician may not refer the patient to the surgeon because of concerns over increased risk due to progressed lung disease, cardiac or other comorbidities. Previous studies have suggested that the proportion of patients who remain undiagnosed and clinically categorized as unclassifiable fibrosis may range from $12-30 \%(4,5)$.

\section{What is the role of transbronchial cryobiopsy?}

Transbronchial cryobiopsy (CryoTBB) is a new minimal invasive tool for bronchoscopic lung tissue sampling. CryoTBB samples are larger than biopsies sampled by transbronchial forceps biopsy and show no crush artifacts $(6,7)$.

Early metaanalyses on CryoTBB have shown a high diagnostic yield with a pooled estimate of $80-85 \%(8,9)$. CryoTBB and SLB have been shown to have a comparable influence on MDD (10). However, the diagnostic confidence of the pathologists was lower for CryoTBB than for SLB in this study.

It has been shown that morbidity and mortality after CryoTBB may be lower than after SLB $(1,11)$. However, morbidity and mortality after CryoTBB may be higher than reported in the early studies (12).

Overall, the role of CryoTBB still remains unclear. Due 
to the low evidence, the guidelines formally do not give a recommendation on the use of CryoTBB (1).

Under the assumption of a lower morbidity and mortality according to recent data, CryoTBB could represent an interesting minimal invasive diagnostic procedure which may dispense of SLB in a relevant proportion of patients.

In a previous prospective study of 61 subjects, it could be shown that the diagnostic confidence of histopathological findings after CryoTBB was high (definite or probable) in about $67 \%$ of cases and lead to a conclusive diagnosis after MDD in $77 \%$ of cases. In the cases where the diagnosis remained unclear, a step-up SLB procedure had an additional diagnostic impact at the MDD in about 23\% (13).

These and other encouraging data showed the need for a study that determines the diagnostic accuracy of the procedure.

\section{What does COLDICE add to the field? What are the limitations of the study?}

Recently, a prospective comparative multicenter study (COLDICE) has been published which aimed to determine the diagnostic accuracy of CryoTBB (14).

65 ILD subjects with the indication for lung tissue sampling were enrolled within a time period of approximately three years at nine Australian tertiary care hospitals (48\% men, mean age 66.1 years). Mean forced vital capacity was $83.7 \%$ of predicted value (\% pred) with a standard deviation (SD) of 14.2. Mean diffusion capacity of the lungs for carbon monoxide was $63.4 \%$ pred (SD 12.8). A bronchoscopic CryoTBB procedure followed by a SLB procedure were performed sequentially in every subject under general anesthesia. Pseudonymized samples were analyzed by blinded pathologists. In the MDD, pseudonymized cases were evaluated twice with either CryoTBB or SLB results added to the discussion in a randomized non-consecutive order.

It was aim of the study to determine the agreement of histopathological results derived from CryoTBB and SLB and of consensus clinical diagnosis using CryoTBB and SLB results at MDD.

Troy et al. showed that the agreement for paired CryoTBB and SLB for guideline-refined histopathological patterns was $70.8 \%$ (kappa 0.70, 95\% CI: 0.55-0.86). Agreement for the specific histopathological patterns was 69.2\% (kappa 0.47, 95\% CI: 0.30-0.64). Interestingly, no procedure-related variables (number, size, site of biopsies, freezing time) were associated with the agreement of
CryoTBB and SLB. This is in accordance with previous data (13). However, in the COLDICE study the number of subjects was too low to allow concise statistical calculations regarding the influence of procedure-related variables. For histopathology interpretation, the interrater agreement was moderate for CryoTBB (kappa 0.52, 95\% CI: 0.44-0.60) and substantial for SLB (kappa 0.64, 95\% CI: 0.54-0.75).

High confidence or definite final MDD diagnosis was reached in $60 \%$ of CryoTBB cases (74\% of SLB cases; $\mathrm{P}=0.090)$. In these Cryo-TBB cases the concordance with SLB results reached $95 \%$. This demonstrates clearly that high confidence or definite final MDD diagnosis based on CryoTBB results are reliable. The addition of CryoTBB histopathology to the MDD was helpful in $74 \%$ of cases. In cases with unclassifiable or low confidence CryoTBB MDD diagnosis, $23 \%$ of cases were reclassified into alternative high confidence or definite MDD diagnosis by SLB.

These results confirm the results of a previous prospective study, where a high confidence or definite final MDD diagnosis was reached in $77 \%$ of Cryo TBB cases. $23 \%$ of unclassifiable or low confidence CryoTBB MDD diagnoses were reclassified into alternative high confidence or definite MDD diagnosis by SLB (13). In this study the interrater agreement was good (kappa 0.73, 95\% CI: 0.64 0.81 ) and intrarater-agreement was excellent (kappa 0.85, 95\% CI: 0.71-0.99).

The novel and outstanding aspect of the COLDICE study is the attempt to determine the diagnostic accuracy. Although the number of included subjects is low, the multicenter approach and the concise study design lead to robust study results that are consistent with previous studies.

Analyzing the study in detail, some data may induce further discussion and interpretation.

CryoTBB samples are smaller than SLB samples and mostly do not include pleura (only $11 \%$ of CryoTBB samples in the COLDICE study). In the past, these considerations lead to the hypothesis that histopathological information from CryoTBB samples may be helpful but on the whole inferior to SLB samples. This is in consistency with the findings of Tomassetti et al. They showed in their previous study that the histopathological information from CryoTBB and SLB specimens had a comparable influence on the MDD, however the diagnostic confidence of the pathologists were lower for CryoTBB specimens (10). The published COLDICE data deliver only limited information about the diagnostic confidence of the pathologists. A grading of diagnostic confidence 
is not reported for the specific histopathological pattern of CryoTBB and SLB specimens (supplementary table 3 of the original publication). Comparing the guidelinereferred histopathological patterns for UIP, the finding definite UIP is reported in 10 CryoTBB and 27 SLB cases, whereas probable UIP is reported in 27 CryoTBB and 6 SLB cases (figure 3 of the original publication). Frequencies for the categories "indeterminate for UIP" and "alternative diagnosis" were nearly equal between both procedures. These findings support the hypothesis that histopathological findings from CryoTBB samples may be characterized by a lower confidence. Given clinically suspected IPF and radiologically confirmed definite UIP, probable UIP or indeterminate for UIP both histopathological patterns, definite UIP and probable UIP, lead to the MDD diagnosis "IPF" or "IPF (likely)" (1). These considerations demonstrate, that in suspected IPF CryoTBB and SLB may be of comparable diagnostic value in the MDD, supporting the results of Tomassetti et al. (10).

The authors report the histopathological patterns and MDD diagnosis for CryoTBB and SLB specimens (Table 1). Analyzing the data for both procedures, it has to be noted that there are 38 CryoTBB cases and 35 SLB cases of IPF, respectively, and 15/18 cases of hypersensitivity pneumonitis (HP) but only one MDD diagnosis of idiopathic nonspecific pneumonia (iNSIP) in the whole cohort. This is striking and different from observations in previous studies.

Apart from HP, iNSIP represents one of the most important differential diagnoses of IPF. MDD is essential to establish the clinical diagnosis of iNSIP and to differentiate it from IPF. This is important as both entities, iNSIP and IPF, belong to the subgroup of chronic fibrosing idiopathic interstitial pneumonitis (15). In contrast to IPF, there is no evidence-based consensus NSIP guideline for a standardized diagnostic algorithm. Clinical features of discordant cases at MDD are given in the supplementary table 4 of the original publication. The reported discordance results from different MDD diagnoses based on Cryo TBB and SLB findings in 15 cases. In 12 out of these 15 cases the discordant diagnoses are IPF versus HP versus unclassifiable fibrosis. Clinically relevant exposure is reported only in 7 out of these 15 cases. This may lead to the hypothesis that HP may be over- and iNSIP may be underrepresented in the MDD diagnoses of the COLDICE study.

NSIP is the typical histopathological pattern of the clinical diagnosis iNSIP. In the study, there are only three cases reported with a histopathological NSIP pattern. It has to be pointed out, that the histopathological NSIP pattern occurs not only as an idiopathic condition, but also in a variety of settings including connective tissue disease, HP and drug induced pneumonitis, and in some patients with familial pulmonary fibrosis. The reported three cases were diagnosed as connective tissue disease related ILD $(n=1)$, $\mathrm{HP}(\mathrm{n}=1)$ and $\operatorname{iNSIP}(\mathrm{n}=1)$.

SLB samples are larger and include representative material of the visceral pleura and adjacent subpleural tissue. It may be hypothesized that interrater agreement may be higher for SLB samples. The interrater-agreement in this study was only moderate for both techniques, CryoTBB and SLB. This is in accordance with previous studies and demonstrates clearly that the pathological evaluation of ILD specimens is difficult and that the findings may be influenced by subjective variables as standardization is low.

In one case, MDD lead to the clinical diagnosis of lymphangioleiomyomatosis (LAM). This is surprising as none of the CryoTBB or SLB samples described a LAM pattern. The clinical diagnosis of LAM based on clinical, radiological and blood-derived vascular endothelial growth factor findings seems reasonable in some cases but is questionable when there is no correlate in histopathological specimens.

Due to the design, the study does not deliver comprehensive data on complication rates of each procedure. Overall, there are no signs that may lead to the suspicion of elevated rates of bleeding or mortality when compared to previous studies.

\section{Conclusions and future directions}

The COLDICE study adds essential evidence to the scientific field. The reported CryoTBB data can be used to estimate the diagnostic accuracy and the potential role of CryoTBB in clinical practice. Through the comparison of CryoTBB with SLB, much has been learned about the reliability of CryoTBB results. Larger multicenter studies may further substantiate the data and help to determine the precise diagnostic accuracy. However, ethical concerns may hinder both procedures being carried out in all subjects when considering procedure-associated risks of CryoTBB and SLB (COLDICE: 90-day mortality 2\%). As the results of this study are convincing, COLDICE may remain the only well-designed study where both procedures are performed in all subjects.

The results of COLDICE are encouraging and show clearly that CryoTBB results are reliable in cases where CryoTBB lead to high confidence or definite final 
Table 1 Histopathological patterns and multidisciplinary discussion diagnosis for specimens (modified) (14)

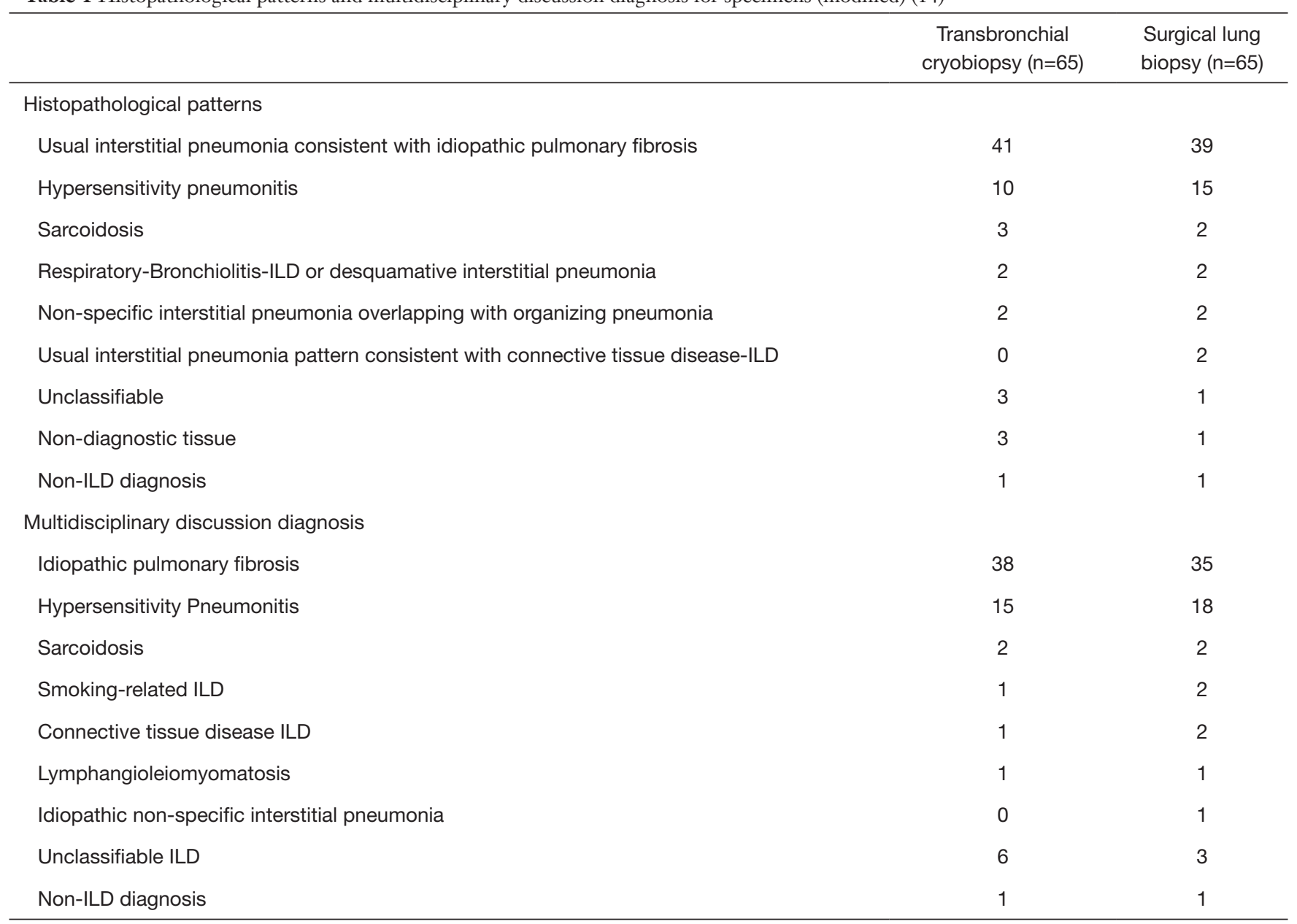

ILD, interstitial lung disease.

MDD diagnosis. These data are well in accordance with previous data and may lead to a revision of the guideline recommendations regarding the role of CryoTBB in the diagnostic algorithm of fibrosing interstitial lung disease.

\section{Acknowledgments}

Funding: None.

\section{Footnote}

Provenance and Peer Review: This article was commissioned by the editorial office, Annals of Translational Medicine. The article did not undergo external peer review.

Conflicts of Interest: All authors have completed the ICMJE uniform disclosure form (available at http://dx.doi. org/10.21037/atm-20-3132). The authors have no conflicts of interest to declare.

Ethical Statement: The authors are accountable for all aspects of the work in ensuring that questions related to the accuracy or integrity of any part of the work are appropriately investigated and resolved.

Open Access Statement: This is an Open Access article distributed in accordance with the Creative Commons Attribution-NonCommercial-NoDerivs 4.0 International License (CC BY-NC-ND 4.0), which permits the noncommercial replication and distribution of the article with the strict proviso that no changes or edits are made and the original work is properly cited (including links to both the 
formal publication through the relevant DOI and the license). See: https://creativecommons.org/licenses/by-nc-nd/4.0/.

\section{References}

1. Raghu G, Remy-Jardin M, Myers JL, et al. Diagnosis of Idiopathic Pulmonary Fibrosis. An Official ATS/ERS/JRS/ ALAT Clinical Practice Guideline. Am J Respir Crit Care Med 2018;198:e44-68.

2. Flaherty KR, King TE Jr, Raghu G, et al. Idiopathic interstitial pneumonia: what is the effect of a multidisciplinary approach to diagnosis? Am J Respir Crit Care Med 2004;170:904-10.

3. Flaherty KR, Andrei AC, King TE, Jr., et al. Idiopathic interstitial pneumonia: do community and academic physicians agree on diagnosis? Am J Respir Crit Care Med 2007;175:1054-60.

4. Guler SA, Ellison K, Algamdi M, et al. Heterogeneity in Unclassifiable Interstitial Lung Disease. A Systematic Review and Meta-Analysis. Ann Am Thorac Soc 2018;15:854-63.

5. Guenther A, Krauss E, Tello S, et al. The European IPF registry (eurIPFreg): baseline characteristics and survival of patients with idiopathic pulmonary fibrosis. Respir Res 2018;19:141.

6. Franke KJ, Linzenbold W, Nuessle D, et al. A New Tool for Transbronchial Cryobiopsies in the Lung: An Experimental Feasibility ex vivo Study. Respiration 2016;91:228-34.

7. Pajares V, Puzo C, Castillo D, et al. Diagnostic yield of transbronchial cryobiopsy in interstitial lung disease: a randomized trial. Respirology 2014;19:900-6.

8. Johannson KA, Marcoux VS, Ronksley PE, et al. Diagnostic Yield and Complications of Transbronchial

Cite this article as: Hagmeyer L, Matthes S, Frank K, Randerath W. Diagnosis in interstitial lung disease: highly confident histopathological results from transbronchial cryobiopsy are reliable. Ann Transl Med 2020;8(20):1328. doi: 10.21037/atm-20-3132
Lung Cryobiopsy for Interstitial Lung Disease. A Systematic Review and Metaanalysis. Ann Am Thorac Soc 2016;13:1828-38.

9. Iftikhar IH, Alghothani L, Sardi A, et al. Transbronchial Lung Cryobiopsy and Video-assisted Thoracoscopic Lung Biopsy in the Diagnosis of Diffuse Parenchymal Lung Disease. A Meta-analysis of Diagnostic Test Accuracy. Ann Am Thorac Soc 2017;14:1197-211.

10. Tomassetti S, Wells AU, Costabel U, et al. Bronchoscopic Lung Cryobiopsy Increases Diagnostic Confidence in the Multidisciplinary Diagnosis of Idiopathic Pulmonary Fibrosis. Am J Respir Crit Care Med 2016;193:745-52.

11. Hagmeyer L, Theegarten D, Treml M, et al. Validation of transbronchial cryobiopsy in interstitial lung disease interim analysis of a prospective trial and critical review of the literature. Sarcoidosis Vasc Diffuse Lung Dis 2016;33:2-9.

12. Pannu J, Roller LJ, Maldonado F, et al. Transbronchial cryobiopsy for diffuse parenchymal lung disease: 30- and 90-day mortality. Eur Respir J 2019;54. doi: 10.1183/13993003.00337-2019.

13. Hagmeyer L, Theegarten D, Wohlschlager J, et al. Transbronchial cryobiopsy in fibrosing interstitial lung disease: modifications of the procedure lead to risk reduction. Thorax 2019;74:711-4.

14. Troy LK, Grainge C, Corte TJ, et al. Diagnostic accuracy of transbronchial lung cryobiopsy for interstitial lung disease diagnosis (COLDICE): a prospective, comparative study. Lancet Respir Med 2020;8:171-81.

15. Travis WD, Costabel U, Hansell DM, et al. An official American Thoracic Society/European Respiratory Society statement: Update of the international multidisciplinary classification of the idiopathic interstitial pneumonias. Am J Respir Crit Care Med 2013;188:733-48. 